purity, the Harvard team was able to achieve a sensitivity of $0.5 \mu \mathrm{T}$ per $\mathrm{Hz}^{1 / 2}$. If one assumes that such a nanodiamond (or rather the defect inside) can be brought to within $30 \mathrm{~nm}$ of a sample, this sensitivity is roughly equivalent to $7 \mathrm{Bohr}$ magnetons per $\mathrm{Hz}^{1 / 2}$, which is sufficient to detect the magnetic moment of about seven electron spins in $1 \mathrm{~s}$ (see Fig. 1). This level of sensitivity is almost within a factor of ten of the current capability of nanomechanical force sensors, the most sensitive general-purpose spin detection method available today ${ }^{4}$. Given the proof-of-principle character of the diamond magnetometry experiments, there is certainly considerable scope for future improvement. Indeed, pushing the sensitivity below the single-spin level could pave the way for the magnetometer to be used as a quantum read-out device, which would make possible a variety of quantum measurement experiments.

By attaching a tiny diamond to the tip of the cantilever in an atomic force microscope, Fedor Jelezko of the University of Stuttgart and co-workers ${ }^{2}$ also demonstrate that it is possible to perform nanoscale magnetic imaging with a single spin. In their experiment, they map the magnetic field of a small ferromagnetic test structure, achieving a spatial resolution of about $20 \mathrm{~nm}$.

Significantly, the resolution in this approach is restricted only by the magnetic sensitivity of the spin and the field gradient of the ferromagnet, not the size of the atomic-scale diamond defect.

To image on the scale of individual atoms and molecules, it will be necessary to improve the sensitivity and resolution of diamond magnetometers by several orders of magnitude. For this purpose, the separation between the diamond spin and the sample must be reduced and the spin lifetime must be increased - meaning smaller, purer nanocrystals. Ideally, only a single nitrogen-vacancy defect should be present in a perfect single crystal, so methods for the controlled implantation of single nitrogen impurities into ultrapure crystals are crucial. It is also an open question how much the size of nanodiamonds can be reduced, and how close to a surface the defects can be placed, without corrupting their magnetic sensing capability.

Finally, diamond sensors may end up being useful for an entirely different application. Given that the single spin can map unknown magnetic fields with nanometre spatial resolution, what about the opposite? Can one use a known magnetic field distribution to locate a diamond nanoparticle precisely? In a proof-of-principle experiment Jelezko and co-workers demonstrate the potential of this approach for high-resolution optical microscopy. By scanning a nanoscale magnetic tip over an immobilized nanodiamond, they are able to locate the position of the particle with nanometre precision. They also show that two diamond spins roughly $100 \mathrm{~nm}$ apart can be resolved with an accuracy of $20 \mathrm{~nm}$ - well below the diffraction limit of optical microscopy, which is at about $200 \mathrm{~nm}$ (see the Supplementary Information to ref. 2). When used as fluorescent biomarkers in cells, nanodiamonds could enable similar improvements to be made to the resolution of optical microscopy.
Several avenues for extending fluorescence microscopy to the nanoscale are currently being pursued but they will require substantial innovation to reach the molecular level ${ }^{8,9}$. For nanodiamonds the resolution is set by the magnetic field sensitivity, not by the optical properties of marker molecules. As the Stuttgart team points out, nanometre resolution can be achieved without any probes located closer than typical cell dimensions. Critically, diamond nanoparticles are also very well suited for in vivo experiments - they are non-toxic and it is easy to modify their surface chemistry for targeted applications in biology.

Defects in diamond have attracted interest from a variety of disparate fields, such as spintronics, materials science and bioimaging, and it is exciting to see how diamond magnetometry brings all these efforts together to create a novel field of research that will, in turn, open up new possibilities for all of them.

References

1. Maze, J. R. et al. Nature 455, 644-647 (2008),

2. Balasubramanian, G. et al. Nature 455, 648-651 (2008).

3. Rugar, D., Budakian, R., Mamin, H. J. \& Chui, B. W. Nature 430, 329-332 (2004).

4. Degen, C. L. et al. Presented at 49th Experimental NMR Conference, Asilomar, California, 9-14 March 2008; $<$ http://enc-conference.org/longabs/E081130.0000VER.1.pdf>.

5. Chernobrod, B. M. \& Berman, G. P. J. Appl. Phys. 97, 014903 (2005).

6. Degen, C. L. Appl. Phys. Lett. 92, 243111 (2008).

7. Taylor, J. M. et al. Nature Phys. 4, 810-816 (2008).

8. Willig, K. I., Rizzoli, S. O., Westphal, V., Jahn, R. \& Hell, S. W. Nature 440, 935-938 (2006).

9. Betzig, E. et al. Science 313, 1642-1645 (2006)

10. Boero, G., Demierre, M., Besse, P. A. \& Popovic, R. S. Sens. Actuat. A 106, 314-320 (2003).

11. Kirtley, J. R. et al. Appl. Phys. Lett. 66, 1138-1140 (1995).

12. Gardner, B. W. et al. Rev. Sci. Instrum. 72, 2361-2364 (2001)

13. Huber, M. E. et al. Rev. Sci. Instrum. 79, 053704 (2008).

\title{
MOLECULARSWTCHES
}

\section{Order and control}

Molecules that can reversibly switch between at least two states in response to an external trigger are essential for the development of molecular electronics. Azobenzene - a molecule composed of two phenyl rings linked by a nitrogennitrogen double bond - has been of particular interest in this regard because it can reversibly isomerize between trans and cis forms under the influence of light, an electric field or tunnelling electrons. However, future devices will require functional molecules, integrated into larger architectures that can be addressed selectively. Now Stefan Hecht of Humboldt University in Berlin, Leonhard Grill of the Free University

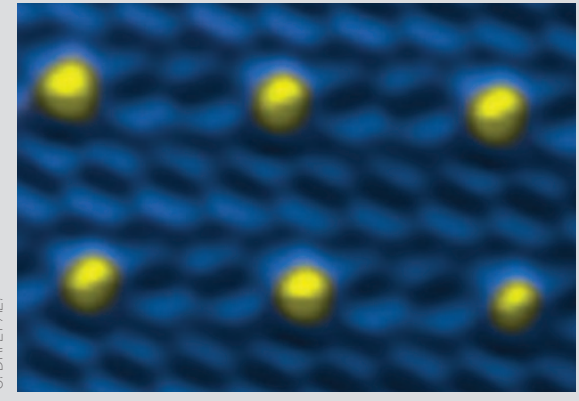

of Berlin and colleagues have shown that molecules can be collectively switched with spatial selectivity (page 649).

The researchers used an asymmetric azobenzene derivative containing a single methoxy group $\left(-\mathrm{OCH}_{3}\right)$, which was adsorbed on a gold surface and examined with a scanning tunnelling microscope (STM). Voltage pulses from the STM tip were used to transform the molecules from the relatively flat trans configuration to the three-dimensional cis configuration. The team found that the probability of switching was significantly influenced by the surrounding molecules and supporting surface. As a result, they were able to create the same lattices of cis isomers in repeated switching cycles. The image here shows a STM image of such a lattice, with the cis isomers in yellow surrounded by the trans isomers in blue.

Owain Vaughan 